\title{
Quality Evaluation and Automatic Classification in Resistance Spot Welding by Analyzing the Weld Image on Metal Bands by Computer Vision
}

\author{
Yang ou ${ }^{1,2,3}$ and Li yueping ${ }^{1}$ \\ ${ }^{1}$ Department of Applied Computer Engineering, Shenzhen Polytechnic \\ ${ }^{2}$ key laboratory of optoelectronic devices and system of ministry of education and \\ Guangdong province \\ ${ }^{3}$ School of Information Engineering Changan University \\ yangou@szpt.edu.cn
}

\begin{abstract}
This paper introduces a novel method for the quality evaluation of resistance spot welds. The evaluation is based on computer vision methods, which allow nondestructive on-line real-time processing. The input of the system is the image of a weld imprint on a metal band which covers the electrodes against wear and soiling. In order to find the position of the resistance spot welds, we describe an image registration method based on geometric pattern matching for alignment system in metal parts. Further we extract features describing the shape of localized objects in segmented images. Using these shape descriptors (geometric feature) we classify the defects by Artificial Neural Network.
\end{abstract}

Keywords: Artificial Neural Network, spot weld, computer vision, Image features extraction, image Segmentation.

\section{Introduction}

Machine vision is one of key technologies in manufacturing because of in increasing demands on the documentation of quality and the traceability of products. It is concerned with engineering systems, such as machines or production lines, that can perform quality inspections in order to remove defective products from production or that control machines in other ways, e.g., by guiding a robot during the assembly of a product. Some of the common tasks that must be solved in machine vision system are as follow: object identification is used to discern different kinds of objects, e.g., character strings or bar codes, or on specific characteristics of the objects themselves, such as their shape [1-4]; position detection is used, for example a pick-and-place machine that places electronic components onto a printed circuit board (PCB), position detection can be performed in two or three dimensions, depending on the requirements of the application [5-8]; surface inspection is used to check the surface of a finished product for imperfections such as scratches, indentations, protrusions, etc., a ball grid array (BGA) is a chip package having solder balls on the underside for mounting on a circuit board [9], to ensure a proper connection to the circuit board, it is important that all individual balls are at the correct position, have the correct size, and are not damaged in any way, in this application, the size and shape of the balls are checked, the BGA is tested for missing or extraneous balls as well as for wrongly positioned balls. The algorithms used in these applications are: thresholding, extraction of connected components, region features, subpixel-precise thresholding, contour features, edge extraction, geometric transformations, region morphology, robust template matching, pattern recognition and classification.

For several decades, resistance spot welding has been an important process in sheet metal fabrication. The automotive industry, for example, is dominated by spot welding, 
due to its simple and cheap operation. The advantages of spot welding are numerous and include the following: an economical process, adaptable to a wide variety of materials (including low-carbon steel, stain-less steel, aluminum, nickel, titanium, and copper alloys) and thicknesses, a process with short cycle times, and a relatively robust process with some tolerance to fit-up variations. However, given the uncertainty associated with individual weld quality (attributed to factors such as tip wear, sheet metal surface debris, fluctuations in power supply, etc.,) it is a common practice in the industry to add a significant number of redundant welds to gain confidence in the structural integrity of the welded assembly. In recent years, global competition for improved productivity and reduced non-value-added activity is forcing companies such as the automotive OEMs to eliminate these redundant spot welds. In order to minimize the number of spot welds and, yet, still satisfy essential properties such as strength, weld quality must be obtained [1012].

Resistance spot welding is a commonly used sheet metal joining process in a number of industries, chiefly, in the automotive industry. One priority of these industries is the production of high-quality spot welds to ensure the stability and safety of their products. One way to produce high-quality spot weld joints is to monitor the welding parameters, such as voltage, welding current, electrode pressure and ultrasonic transmission.

But any kind of parameter monitoring can only provide an indirect estimation of the welding quality, which does not lead to reliable estimations of the weld nugget size. It cannot ensure the weld quality by directly measuring the nuggets.

Our goal is to go beyond parameter monitoring and to provide a fully automatic quality inspection of resistance spot welds using image-processing techniques. The quality estimation of the spot weld joints is based on the segmentation and measurement of weld imprints on metal bands.

The following sections present related work by scholars in this field, Section 2, 3 explain proposed algorithm with experimental methodology .It also covers acquisition of images, image pre-processing, image registration, image template matching, weld feature extraction $[13,14]$. Section 4 describes classification and results respectively.

\section{SYSTEM OVERVIEW AND RELATED WORK}

The graph and photo of the overall inspection systems is shown in Figure 1 and Figure 2 , the machine vision systems is used to capture the images. Vision -based inspection systems are a set of new technologies for non-contact inspections and measurements. The instruments integrate a multitude of technologies including digital imaging, electronics, embedded systems and software. In this proposed vision system, images of welding surfaces are captured through a CCD camera. The CCD image sensor is a monochromatic sensor chip with a $1392 * 1040$ array of 8 -bit pixels, it can be used to acquire gray scale image with a light intensity ranging from 0 to 255 , while 0 represents the lowest light intensity, and 255 represents the highest light intensity. The frame rate of the selected GigE camera is up to 100 frames per second, and the cable length is up to $100 \mathrm{~m}$. In front of the camera, a lens $(20 \mathrm{~mm})$ is also precisely arranged in a designed distance Images are obtained using red coaxial parallel lights which adopt special optical lens group to achieve illuminating the product surface vertically and uniformly; therefore clearly highlight bump defects on the flat surface.

In computer vision, it is essential to create constant and well defined lighting conditions if possible. Well defined and homogeneous illumination improves the reliability of subsequent image-processing algorithms. For homogeneous illumination of the electrode band, we use eight white circularly arranged light-emitting diodes (LEDs) which are enclosed in a dome-shaped case, as shown in Figure 3. This construction nearly prevents the influence of ambient light and ensures constant homogeneous lighting conditions. 


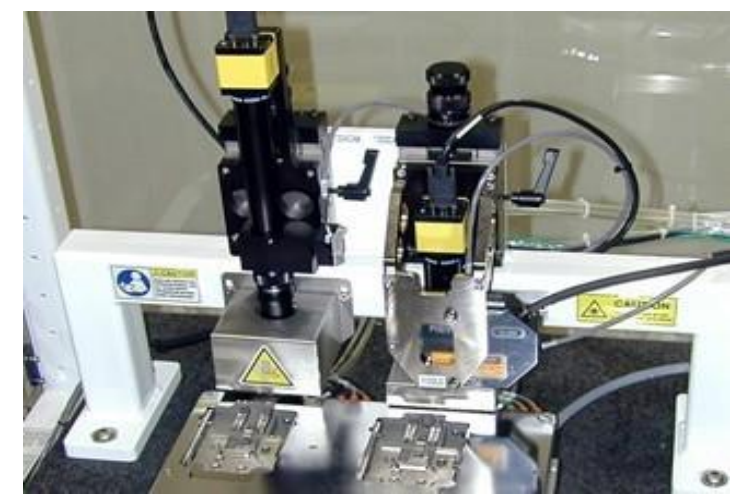

Figure 1. Graph of the Machine Vision System

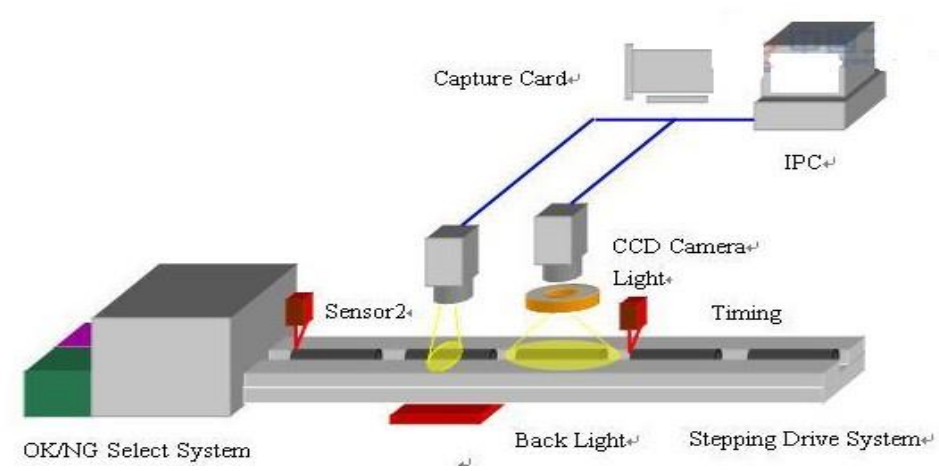

Figure 2. Photo of the Machine Vision System

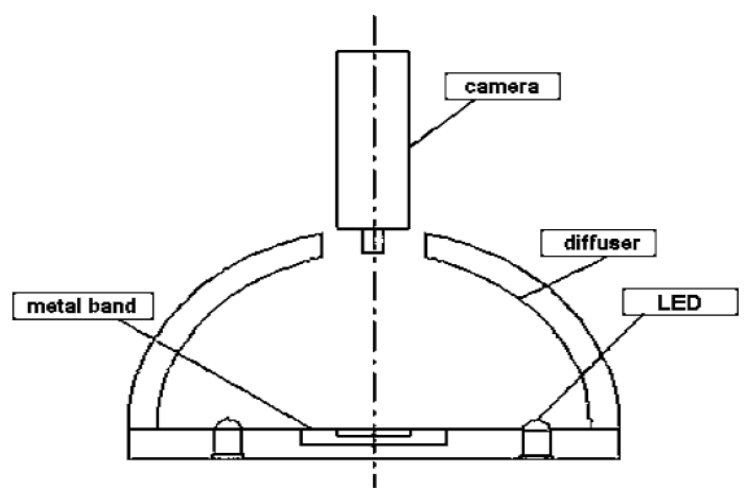

\section{Figure 3. Profile of the Principal Setup of the Illumination System using a Dome-shaped Case, Together with Eight Light-emitting Diodes (LEDs)}

The following section introduces related work. Many of the authors/developers suggest extraction of weld seam from the radiographic images when looking for the welding defects. The reason presented for this step is quite obvious, as defects are present only in weld seam which are to be detected and classified later. Extracting weld seam provides a form of ROI (region of interest) in the radiographic images. Liao and Ni10 proposed an algorithm for extracting weld seam. The scheme was developed by observing the intensity plots in which weld seam looks more like Gaussian than other objects present in the image. Similarity measure between an object and its intensity plot is defined in terms of MSE (mean square error). The concept behind this method is: value of MSE (for objects present in image) is inversely proportional to the Gaussian nature of the object. So lower the value of MSE; the object tends to be more Gaussian in nature. Hence object with the 
lowest MSE is chosen to be weld seam. Another approach was presented by Mahmoudi and Regragui. They proposed to perform global thresholding using otsu's method after performing homomorphic filtering. Homomorphic filtering normalizes brightness and increases contrast simultaneously [12]. Wang, et al., devised another technique for weld seam extraction [18]. The technique is based on intensity values of different areas present in the image. Weld image is classified into three different areas, i.e., base metal area, the weld area and lead plate area. Base metal appears brighter than both weld area and lead plate area. The lead plate area is the darkest area in the image. Binary image is automatically computed from gray level histogram. Adaptive segmentation is used, which works on the principle that variance inside the class is least while outside of class is the largest one. In a system was proposed for automatic identification of weld defects using digital image processing, feature extraction and pattern classification. Image processing techniques of noise reduction and contrast enhancement were used along with BSM (background subtraction method) for segmentation [13, 14]. Features describing the shape, size, intensity and location of welding defects, distance from centre, standard deviation, major axis, width and length, elongation, hey wood diameter, average intensity and standard deviation of intensity were used. Pattern classification was carried out using two techniques namely fuzzy k-NN and MLP neural network. Shafeek, et al., proposed another technique using software support. AutoWDI (automatic welding defect inspection) and AutoWDA (automatic welding defect assessment) were used as primitive tools for automatic inspection of weld defects in gas pipelines. Form factor and regularity factor were used for defect identification which was further used for final detection of defects. One more method for detection of weld defects using multiple thresholds was proposed in [14]. They proposed a method using Hough transform to remove noisy pixels in coarse defect region. Features extraction was done with multiple thresholds. Features extraction was followed by SVM (support vector machine) to classify defects 15, 16. Some suitable features were used in their technique to estimate accuracy of classification of weld defects. Position, ratio of aspect, roundness, area and angle were used for estimating the accuracy of classification for weld defects by Silva, et al., Some features were normalized prior to the classification. Another approach for defect detection in xray images using fuzzy reasoning was presented by Lashkia. He proposed a technique rich with the special features that uses common sense fuzzy reasoning rules offering an intrinsic understanding of classification logic. Classification is done on basis of visual representation, i.e., thread like zone with low contrast are cracks while roundish zone having high contrast are blow hole defects. Vilar, et al., proposed another system for classification of weld defects in radiographic images [16]. The algorithm starts with preprocessing of images through Wiener and Gaussian filtering. Preprocessing is followed by weld extraction step which mainly uses otsu's method. The last stage is feature extraction; features extracted are area, centroid, major axis, minor axis, eccentricity, euler number, solidity, extent and position. Following features extraction PCA (principal component analysis) is performed to reduce the dimension of input feature vector of classifier. Classification is done using multi-layer feedforward ANN. Valavanis and Kosmopoulos presented another technique for multiclass defect detection and classification in weld radiographic images using geometric and texture features [17]. The paper describes methodology to extract 43 descriptors corresponding to texture measurements and geometrical features for each segmented object and given as input to the classifier. Texture and geometric features allow better modelling of various defects. SBS (sequential backward selection) has been used to avoid computationally intractable exhaustive feature selection. Yahia, et al., proposed another welding defect detection method using radiographic images with neural [18]. This method essentially works on edge detection method based on MPC (multi-layer perceptron). A database of different pictures is prepared with a picture size of $3 \times 3$ which is considered elementary contours. These elementary contours are learned by multi-layer neural network. 48 forms of basic 
contours are used in this paper. Image segmentation is done by thresholding (binarization) by maximizing interclass variance.

\section{EXPERIMENTAL METHODOLOGY}

The images are transferred to PC for further processing by the proposed algorithm. Figure 4 shows the key steps of proposed algorithm. Digital image processing tools are used to de-noise the image collected by CCD camera. Morphological techniques form the basis of this defect detection algorithm. Features like area, major axis length, eccentricity, solidity, convex area, convex hull, position are extracted. Table I shows some of the extracted features. Optimal features are input to artificial neural network. ANN is prior trained for different types of defects classification.

\section{Figure 4. Proposed Algorithms}

\subsection{The Resistance Spot Welding Location}

Feature detection and matching are an essential component of many computer vision applications. Consider the two pairs of comparison images .For the first pair, we may wish to align the two images so that they can be seamlessly stitched into a composite mosaic. For the second pair, we may wish to establish a dense set of correspondences so that a 3D model can be constructed or an in-between view can be generated. The firs kind of feature that you may notice are specific locations in the images, such as mountain peaks, building corners, doorways, or interestingly shaped patches of snow. These kinds of localized feature are often called key point features or interest points (or even corners) and are often described by the appearance of patches of pixels surrounding the point location . Another class of important feature are edges e.g., the profile of mountains against the sky. These kinds of features can be matched based on their orientation and local appearance (edge profiles) and can also be good indicators of object boundaries and occlusion events in image sequences. Edges can be grouped into longer curves and straight line segments, which can be directly matched or analyzed to find vanishing points and hence internal and external camera parameters.

The location and the direction of each part are not the same. Before detecting the spot welding, image registration is performed on the image in order to get both the position and the rotation of the transform. Image registration has many applications, such as manufacturing industry, medicine and military, and it is extensively studied in the past decades. The purpose of image registration is to look for the best transformation which precisely aligns two images by maximizing a similarity measure. There are lots of different image registration methods, which can be classified into two major categories: area-based and feature-based. The method we described in this paper belongs to the latter one. The reason that the edges are used as feature for the registration is not only because edges are hardly affected by illumination changes but also because the computational costs can be reduced. In this paper, we present an accurate and robust image registration method for alignment system in automatic optical inspection (AOI) system for the solder joint inspection.

A) Edge detection and feature description

A digital image is often defined as a two-dimensional discrete function $g(x, y)$, where $x$ and $y$ are spatial coordinates, i.e., position, and $g$ is the intensity. Edges are 
discontinuities of the intensity in an image, and many edge detection methods are based on the derivatives of intensity function $g$.In this paper, the Canny edge detector is employed to obtain the position of edge points with pixel accuracy and the gradient direction of edge points. Let $g_{x}$ be partial derivative in $x$ axis and $g_{y}$ in $y$ axis, the direction of gradient vector is computed by $\arctan \left(g_{y} / g_{x}\right)$. However, edge points with pixel accuracy cannot result in subpixel accuracy image registration. Therefore, an edge detection method that has sub-pixel accuracy is needed. In our present study, a simple sub-pixel edge detection method is used. In this method, for each edge point, one uses the norm of gradient vector of the edge point and those of its two adjacent points in its gradient direction to interpolate a quadratic function, and the position of the extreme is taken as the subpixel position of the edge point.

Although the subpixel edge detection method mentioned above is simple, it is sensitive to noise, thus usually delivering unsmooth edges. In order to remove noises along the edges, the disordered edge points are first connected into chains, then short chains are deleted, and finally geometric de-noising method is applied to each chain to filter the position and direction of the edge points 4,5 . We treat the template image edge points on a chain as a point set of $\mathrm{n}$ points $P=\left\{p_{1}, p_{2}, \ldots, p_{n}\right\}, p_{i} \in R^{2}$, and the subpixel position of edge point $p_{i}$ is denoted as $p_{i}=\left(x_{p i}, y_{p i}\right)$.We use $m$ points to fit a line segment, where $m$ is an odd integer (e.g., 5,7or 9). The $k$-th point is the center of $m$ points, i.e. $k=(m+1) / 2$, and its position and direction are determined by the fitted line (See Figure 3 and 4$)$. The line equations is written as:

$$
\rho=x \cos \theta+y \sin \theta
$$

where $\rho$ is the distance between the origin and the line, and $\theta$ is the angle from $x$ axis to $\rho$ counter clockwise. We use the least-squares method to calculate the parameters of the fitted line. Let $e$ be half of the sum of the square of the distance between each point $p_{i}(i=1,2, \ldots, m)$ and the fitted line

$$
e=\frac{1}{2} \sum_{i=1}^{m}\left(x_{p i} \cos \theta+y_{p i} \sin \theta-\rho\right)^{2}
$$

The minimum of $e$ is achieved where the first-order partial derivatives are zero, that is

$$
\left\{\begin{array}{l}
\frac{\partial e}{\partial \rho}=\sum_{i=1}^{m}\left(x_{p i} \cos \theta+y_{p i} \sin \theta-\rho\right)(-1)=0 \\
\frac{\partial e}{\partial \rho}=\sum_{i=1}^{m}\left(x_{p i} \cos \theta+y_{p i} \sin \theta-\rho\right)\left(-x_{p i} \sin \theta+y_{p i} \cos \theta\right)=0
\end{array}\right.
$$

The solution of equation (3) gives two parameters $\rho$ and $\theta$ of the fitted line.

We project the original $k$-th point onto the fitted line and the position of the projection is considered as the new position of the $k$-th point. The direction of the $k$-th point is corrected at the same time. The difference between the normal direction of the fitted line and the gradient direction of the direction of the fitted line and the gradient direction of the $k$-th point is compared. If the absolute value of the difference point is compared. If the absolute value of the difference is less than a threshold, e.g., 45 degree, the new gradient direction of the $k$-th is set to the normal direction of the fitted line otherwise it is set to the opposite direction. The projected position of the $k$-th point $\left(x_{p k}^{\prime}, y_{p k}^{\prime}\right)$ is given by

$$
\left\{\begin{array}{l}
x_{p k}^{\prime}=\rho \cos \theta+x_{p k} \sin ^{2} \theta-y_{p k} \sin \theta \cos \theta(4) \\
y_{p k}^{\prime}=\rho \sin \theta+y_{p k} \cos ^{2} \theta-x_{p k} \sin \theta \cos \theta
\end{array}\right.
$$



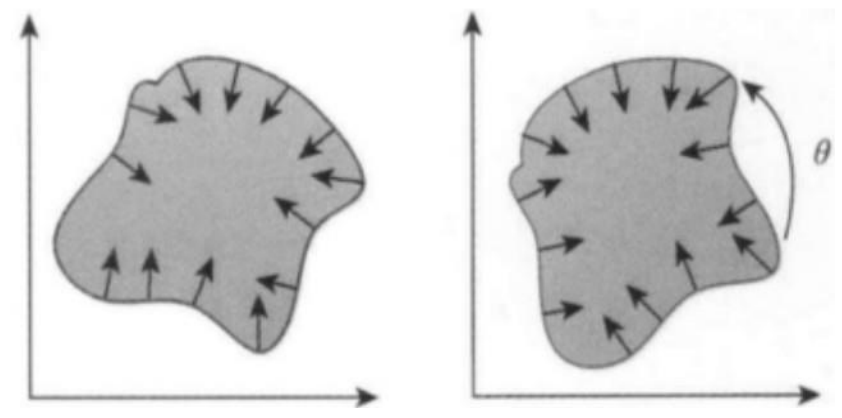

Figure 5. Schematic Diagram of Similarity Measurement

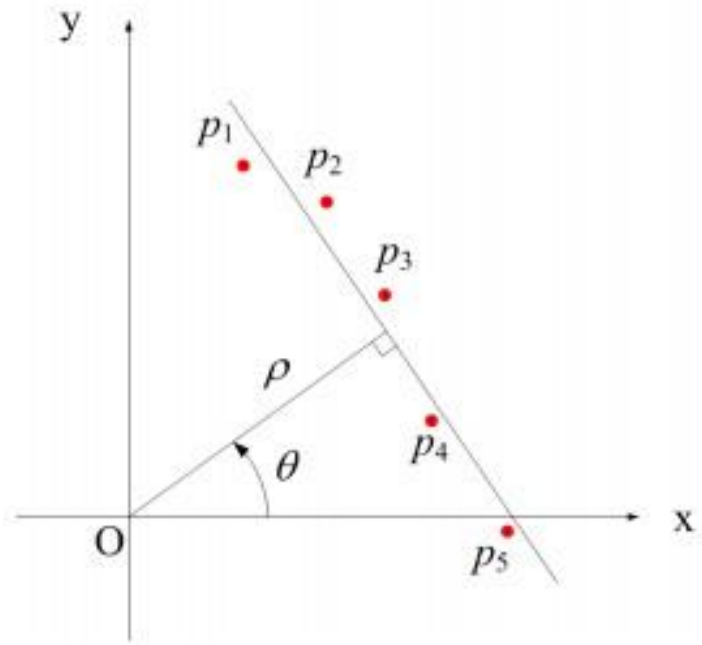

\section{Figure 6. Five Points are used to Fit a Line and the 3rd Point is the Point in Question}

B)Coarse registration

A similarity measure is used for the coarse registration. This similarity measure uses the gradient direction of edge points, and it is proportional to the direction coherence between the template image edge points and the target image edge points. If all point pairs have the same direction, the similarity measure will be 1 , which means completely matching. The similarity measure is defined by 13

$$
s=\frac{1}{n} \sum_{i=1}^{n} \frac{\left\langle g_{p i}^{\prime}, g_{q i}\right\rangle}{\left\|g_{p i}^{\prime}\right\|\left\|g_{q i}\right\|}=\frac{1}{n} \sum_{i=1}^{n} \frac{\left(g_{p i x}^{\prime} g_{q i x}+g_{p i y}^{\prime} g_{q i y}\right)}{\sqrt{g_{p i x}^{\prime 2}+g^{\prime 2}{ }_{p i y}} \sqrt{g_{q i x}^{2}+g_{q i y}^{2}}}
$$

Where $g_{p i}^{\prime}=\left(g_{p i x}^{\prime}, g_{p i y}^{\prime}\right)$ is the gradient of the template edge point $p_{i} \cdot g_{q i}=\left(g_{q i x}, g_{q i y}\right)$ is the gradient of the target edge point $q_{i} \cdot\left\langle g_{p i}^{\prime}, g_{q i}\right\rangle$ is the inner product of $g_{p i}^{\prime}$ and $g_{q i} \cdot\|\bullet\|$ is the L2 norm. 


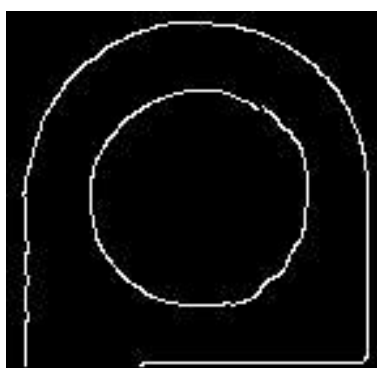

a) edges of template

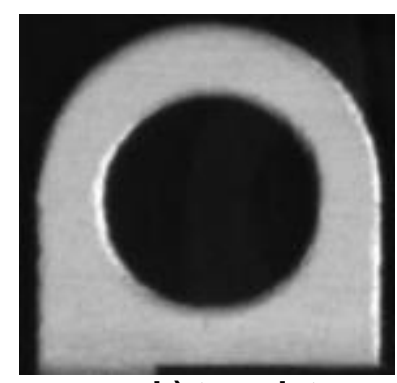

b) template

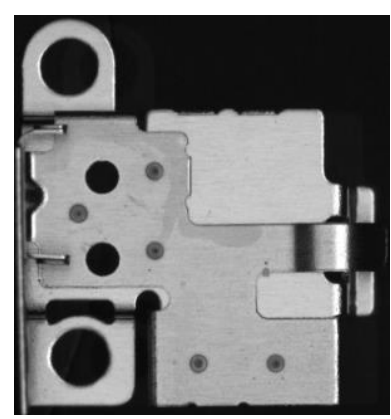

c) metal bands

Figure 7. Edges Extracted from the Template Image in Metal Bands

A template is created by using the edges extracted from the template image (See Figure 7) before carrying out image registration. The template has a pyramid structure. The number of levels of the pyramid should be chosen carefully so that the pattern at the top level is discernible. The pyramid for the target image has the same number of levels as the template image. After the construction of pyramids, a complete searching is performed by using the similarity measure at the top level of pyramid. Because of the small size of the images at the top level, the computational cost is very small. The results obtained at the top level are then used as an initial guess for the searching at the next level. Except at the top level, the searching is performed only in a small window around the results obtained at the higher level. Since the search space is reduced, the coarse registration is efficient.

The results of coarse registration are of pixel accuracy. Four parameters of similarity transformation, i.e., scale parameter $s$, rotation parameter $\alpha$, translation parameters $t_{x}$ and $t_{y}$, between two images are obtained.

\subsection{Segmentation of the Electrode Imprint}

In this proposed vision system, images of welding surfaces are captured through a CCD camera. Using red coaxial parallel lights in this method. From these images, the regions of interest are segmented and the average gray levels of the characteristic features of these images are calculated. During welding process following nine types of flaws are mainly observed. These nine flaws are 1. Slag Inclusion 2. Worm Whole 3. Porosity 4. Incomplete Penetration 5. Under cuts 6. Weaving Fault 7. Cracks 8. Slag Line 9. Lack of Fusion 19.

These Flaws can be detected by various Techniques. One of the well -known Techniques for detection of Flaws is Segmentation. Segmentation is a process in which regions or features sharing similar characteristics are identified and grouped together. Image segmentation is based on threshold, edge detection, region detection or combination of any of these techniques. The segmentation should separate the region that are homogeneous to the particular criteria chosen for analysis, of segmented area should be considerably less than the variation at borders. The best threshold for perfect welding is shape adaptive threshold. As a matter of fact, the welding is approximately circular shaped. Therefore, we can use this knowledge to determine a threshold that lead to the best circular shaped segmentation result. Since a circle has the highest compactness of all 2D shapes, we use the compactness as a feature for the quality of the segmentation image of welding spot. An mathematical definition of the compactness of 2D shapes is given in Eq.6:

$$
\text { Compactness }=4 \pi \frac{\text { Area }}{\text { perimeter }^{2}}
$$

In this algorithm, we first chose high threshold and reduce it iteratively until the results obviously become unacceptable. We calculate the compactness for each of segmentation 
results. Then we analyze the compactness curve to get the highest compactness of the segmentation result which is the most circular shape. We will analyze all kinds of solder joint segmentation image information and a comparative study of qualified and poor solder joint. In Figure 8, a1, b1, c1 and d1 original image of a spot weld imprint a1 and b1 are images of the qualified spot welds. C1and d1 are images of Poor spot welds. a2, b2, c2 and $\mathrm{d} 2$ are the solder joint edge image of inner circle and outer circle. A3, b3, c3 and d3 are segmentation results during the shape adaptive threshold algorithm.

\subsection{Image Features Extraction}

Features are extracted for all the elements are selected on connected component based in segmented images. Features include morphological features, geometric features, color features ,texture features, and statistics features. Geometric features are extracted for all the sections which include area, minor axis length, major axis length, convex area, eccentricity, solidity, convex hull, orientation, extent and extreme, perimeter, centroid, Euler number. These features from the image segmentation are defect candidates so there is a need to discriminate between defects and non-defects. Table 1 show some of the geometric features which are extracted from the segmented images 14, 15, 16.

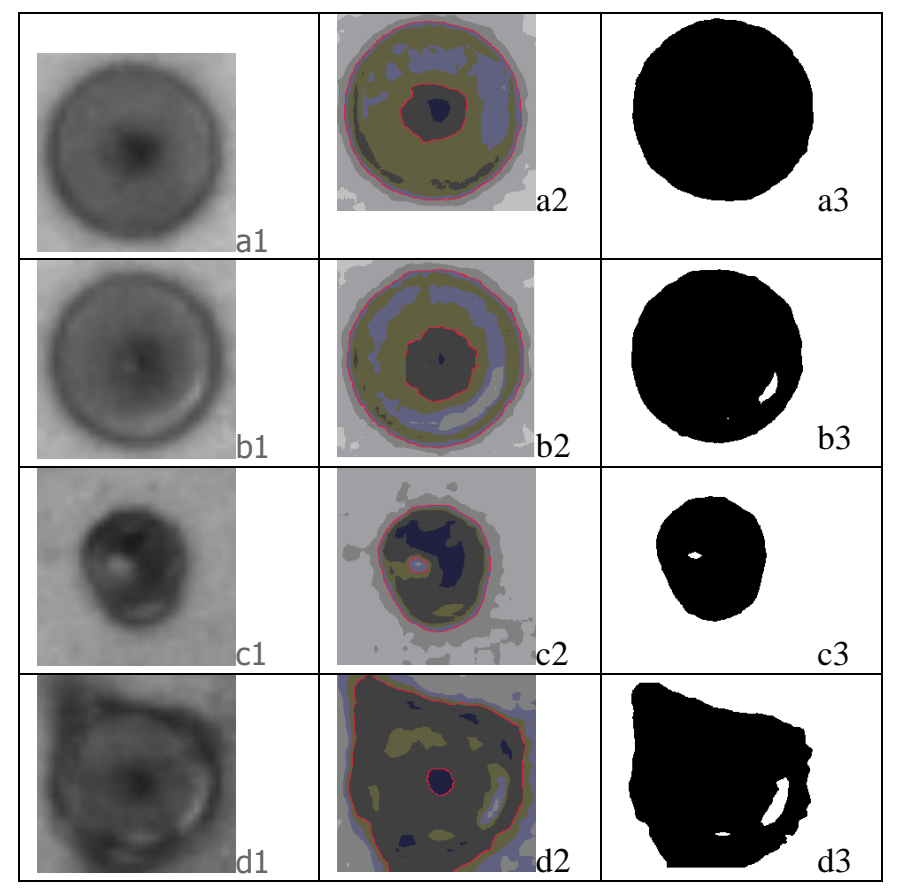

Figure 8. a1, b1, c1 and d1 Original Image of a Spot Weld Imprint. a1 and b1 are Images of the Qualified Spot Welds. C1and d1 are Images of Poor Spot Welds. a2, b2, c2 and d2 are the Solder Joint Edge Image of Inner Circle and Outer Circle. a3, b3, c3 and d3 are Segmentation Results during the Shape Adaptive Threshold Algorithm

Table 1. Geometric Features Extracted For Classification

\begin{tabular}{c|c|c}
\hline \hline No & Name & Notation \\
\hline 1 & Area & $A=\sum_{i=0}^{n} \sum_{j=0}^{n} a_{i, j}$ \\
\hline 2 & Major axis & $L=\sum_{i=0}^{n} \sum_{j=0}^{n} p \cdot\left(a_{i, j}-u\right)$ \\
\hline 3 & Minor axis & $e=\sum_{i=0}^{n} \sum_{j=0}^{n} q \cdot\left(a_{i, j}-u\right)$ \\
\hline
\end{tabular}




\begin{tabular}{c|c|c}
\hline 4 & Solidity & $S=\sum_{i=0}^{n} \sum_{j=0}^{n} a_{i, j} / A_{c}$ \\
\hline 5 & Perimeter & $\begin{array}{c}\mathrm{P}=\text { no. Of pixels forming } \\
\text { boundary of object }\end{array}$ \\
\hline 6 & Convex area & $A_{c}=\sum_{i=0}^{n} \sum_{j=0}^{n} a_{i, j}$ \\
\hline 7 & Eccentricity & $E=\sqrt{1-b^{2} / a^{2}}$ \\
\hline 8 & Orientation & $\mathrm{O}=$ angle of $L$ \\
\hline 9 & Compactness & $\mathrm{C}={ }^{4 \pi \frac{\text { Area }}{\text { perimeter }}}$ \\
\hline \hline
\end{tabular}

\section{AUTOMATIC CLASSIFICATION METHOD TO THE DEFECTS}

\subsection{Classification of Defects using ANN}

We use major axis length, minor axis length, compactness, area and solidity to obtain different defects only from segmented welding image. A multi-layer perceptron (MLP) model using back-propagation (BP) has been used for classification between different defects. The MLP model is shown in Figure 9. The combination of geometry and statistical properties are used for machine learning with machine vision 20, 21.

The general model of MLP consists of a number of nodes arranged in multiple layers with connections between the nodes in the adjacent layers by weights. The model consists of an input layer that accepts the input variables used in the classification, hidden layers and an output layer. A summation of each neuron $j$ in the hidden layer by its input nodes $x_{i}$ after multiplying the connection weights $w_{i j}$ gives the output $y_{j}$ as a function of the sum, that is:

$$
y_{j}=f\left(\sum w_{j i} x_{i}\right)
$$

where $f$ is the sigmoidal or hyperbolic tangent transfer function. Using the BP training algorithm, the weights are minimized based on the squared differences between the actual and desired output values in the output neurons given by:

$$
E=\frac{1}{2} \sum_{j}\left(d_{j}-y_{j}\right)^{2}
$$

where $y_{j}$ is the actual output of the neuron and $d_{j}$ is the desired output of neuron $j$. During classification, input data is fed into the network and the classification is performed by assigning a class number to a pixel or segment using the numerical values computed at the output layer. The weight $w_{i j}$ is updated with an increment $\Delta w_{j i}$ and the error $E$ is reduced until an acceptable value of $E$ is reached. The output node that gives the highest value is set to 1 whereas the others are set to 0 in order to obtain the output class vector 25 , 26.

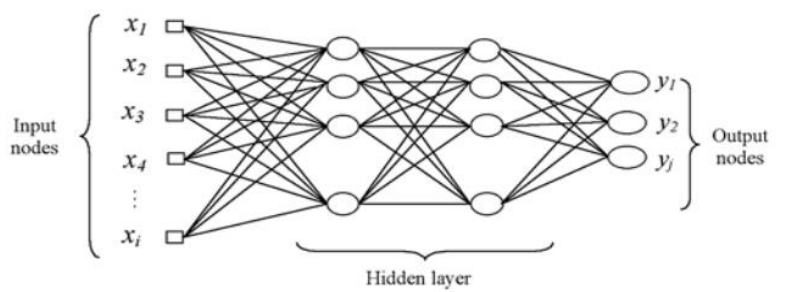

Figure 9. A Schematic of a MLP Model 
Table 2. Results of Image Analysis

\begin{tabular}{|l|l|l|l|l|l|l|l|l|l|l|l|}
\hline & Area & $\begin{array}{l}\text { Major } \\
\text { axis }\end{array}$ & $\begin{array}{l}\text { Minor } \\
\text { axis }\end{array}$ & Solidity & Perimeter & $\begin{array}{l}\text { Convex } \\
\text { area }\end{array}$ & Eccentricity & Orientation & Compacness & $\begin{array}{l}\text { Wel } \\
\text { d } \\
\text { type }\end{array}$ & Result \\
\hline Weld 1 & 542 & 31 & 22 & 0.85 & 85 & 545 & 27 & 59 & 0.77 & 1 & match \\
\hline Weld2 & 587 & 32 & 23 & 0.75 & 88 & 589 & 29 & 42 & 0.80 & 1 & match \\
\hline Weld 3 & 651 & 42 & 20 & 0.80 & 105 & 696 & 27 & 144 & 0.68 & 2 & match \\
\hline Weld 4 & 698 & 42 & 19 & 0.74 & 104 & 731 & 27 & 157 & 0.66 & 2 & match \\
\hline Weld 5 & 145 & 23 & 7 & 0.69 & 84 & 164 & 6 & 64 & 0.65 & 3 & match \\
\hline Weld 6 & 138 & 37 & 4 & 0.85 & 85 & 228 & 4 & 36 & 0.38 & 3 & match \\
\hline Weld 7 & 1235 & 64 & 87 & 0.75 & 160 & 1289 & 29 & 136 & 0.80 & 4 & match \\
\hline Weld 8 & 1249 & 67 & 77 & 0.80 & 176 & 1328 & 27 & 111 & 0.78 & 4 & match \\
\hline Weld 9 & 516 & 169 & 12 & 0.74 & 82 & 523 & 27 & 30 & 0.76 & 5 & match \\
\hline Weld 10 & 517 & 170 & 16 & 0.69 & 84 & 534 & 27 & 88 & 0.73 & 5 & match \\
\hline Weld 11 & 587 & 32 & 23 & 0.75 & 88 & 589 & 29 & 42 & 0.80 & 6 & match \\
\hline Weld 12 & 526 & 29 & 22 & 0.80 & 82 & 528 & 27 & 111 & 0.78 & 6 & match \\
\hline Weld 13 & 516 & 30 & 22 & 0.74 & 82 & 523 & 27 & 30 & 0.76 & 1 & match \\
\hline Weld 14 & 517 & 31 & 20 & 0.69 & 84 & 534 & 27 & 88 & 0.73 & 4 & match \\
\hline
\end{tabular}

\subsection{Results}

From Table 1, the 9 shape descriptors were used to classify the defects. To determine the appropriate input features to train the MLP network, a set of data for training the network using 9 features. In each set, 100 simulated images were used for each defect type, a total of 600 images were used to train 6 defect types. For testing the accuracy of the classification, 60 defect images were used, 10 images for each defect type. The output layer consists of 6 nodes, each node represents a defect type. The network is trained, the accuracy of defect classification reached $98 \% 27$.

\section{Conclusion and discussion}

The spot weld process is a very popular joining mechanism in a number of industries. The quality of the spot weld is very important, since it directly affects the quality of products. Therefore, we need a set of intelligent system capable of detecting the weld quality online. In this paper, we presented an effective and novel method for the quality evaluation of resistance spot welds based on machine vision. The surface image of resistance spot welding joint includes rich quality information of spot weld. Using image segmentation, morphology and particle analysis techniques, the predetermined parameters could be acquired from the weld images. Artificial neural net were used to build the correlation between the acquired parameters and determine the type of defect, the quality of spot welds. For classification we have used nine features from table 1 and used a multilayer perceptron (MLP) model using back-propagation (BP) for classification between different defects. The proposed algorithm is tested on more than 100 images containing various types of detects, the output efficiency of algorithm in detecting and classifying defects is found to be more than $99 \%$ in detecting and $98 \%$ in classifying defects separately.

\section{ACKNOWLEDGEMENTS}

This work is supported by youth foundation of shenzhen Polytechnic (2213K3190016), foundation of shenzhen Polytechnic (2213K3190028) and open foundation of key laboratory of optoelectronic devices and system of ministry of education and guangdong province. 


\section{References}

[1] Q. Huang, M. Li and Z. Ji, "Precisely Locating Multi-QR Code Based on Straight Line Fitting”, Pattern Recognition Chinese Conference (CCPR), (2010) October 15-19, Beijing, China.

[2] Y. Gu and W. Zhang, "QR Code Recognition Based on Image", Processing Information Science and Technology, (2011) March 26-28, Nanjing, China.

[3] H. D. Yuan, "Blind Forensics of Median Filtering in Digital Images", J, Information Forensics and Security, IEEE Transactions on, vol. 6, no. 4, (2011), pp. 1335-1345.

[4] M. R. Farrahi and M. Cheriet, "An Adaptive and Parameter Less Generalization of Otsu's Method for Document Image Binarization”, J. Pattern Recognition, vol. 45, no. 6, (2012), pp. 2419-2431.

[5] P. C. Chang, L. Y. Chen, C.-Y. Fan, "A Case-based Evolutionary Model for Defect Classification of Printed Circuit Board Images", Journal of Intelligent Manufacturing, vol. 19, no. 2, (2008), pp. 203-214.

[6] L. Xie, R. Huang, N. Gu and Z. Cao, "A Novel Defect Detection and Identification Method in Optical Inspection”, Neurual Computing and Applications, vol. 24, no. 6, (2014), pp. 1953-1962.

[7] H. W. Kim and S. I. Yoo, "Defect Detection Using Feature Point Matching for Non-repetitive Patterned Images", Pattern Analysis and Applications, vol. 17, no. 2, (2012), pp. 415-429.

[8] W. Y. Wu, C. W. Hung and W. B. Yu, "The Development of Automated Solder Bump Inspection Using Machine Vision Technoques", The International Journal of Advanced Manufacturing Technology, vol. 69, no. 1-4, (2013), pp. 509-523.

[9] S.-H. Peng and H. D. Nam, "Void Defect Detection in Ball Grid Array X-ray Images Using a New Blob Filter", Journal of Zhejiang University SCIENCE C, vol. 13, no. 11, (2012), pp. 840-849.

[10] T. W. Liao and J. Ni, "An Automated Radiographic NDT System for Weld Inspection: Part I-Weld Extraction”, NDT \& E International, vol. 29, no. 3, (1996), pp. 157-162.

[11] R. R. da Silva, M. H. S. Siqueira, M. P. V. de Souza, J. M. A. Rebello and L. P. Caloba, "Estimated Accuracy of Classification of Defects Detected in Welded Joints by Radiographic Tests", NDT \&E International, vol. 38, (2005), pp. 335-343.

[12] T. Y. Lim, M. M. Ratnam and M. A. Khalid, "Automatic Classification of Weld Defects Using Simulated Data and An MLP Neural Network”, NDT \&E international, vol. 49, (2007), pp. 154-159.

[13] F. Maes, A. Collignon, D. Vandermeulen and G. S. Suetns, "Multimodality Image Registration by Maximization of Mutual Information", IEEE Transactions on Medical Imaging, vol. 16, (1997), pp. 187198.

[14] B. P. F Lelieveldt, R. J. Van der Geest, M. R. Rezaee, J. G. Bosch and J. H. C. Reiber, "Anatomical Model Matching with Fuzzy Implicit Surfaces for Segmentation of Thoracic Volume Scans", IEEE Trans. Med. Imag. vol. 18, (1999), pp. 218-230.

[15] K. Murakami, "Image Processing for Non-Destructive Testing", Welding International, vol. 4, no. 2, (1990), pp. 144-149.

[16] V. Lashkia, "Defect Detection in X-ray Images Using Fuzzy Reasoning", Image and Vision Computing, vol. 19, (2001), pp. 261-269.

[17] K. Xu, A. R. Luxmoore and F. Deravi, "Comparison of Shape Features for the Classification of Wear Particle", Engineering Application of Artificial Intelligence, vol. 10, no. 5, (1997), pp. 485-493.

[18] Y. Wang, Y. Sun, P. Lv and H. Wang, "Detection of Line Weld Detects Based on Multiple Threholds and Support Vector Machine", NDT \& E International, vol. 41, (2008), pp. 517-524.

[19] I. Valavanis and D. Kosmopoulos, "Multiclass Defect Detection and Classification in Weld Radiographic Images Using Geometric and Texture Features", Expert Systems with Applications, vol. 37, (2010, pp. 7606-7614.

[20] N. B Yahia, T. Belhadj, S. Brag and A. Zghal, "Automatic Detection of Welding Defects Using Radiography with a Neural Approach”, Procedia Engineering, vol. 10, (2011), pp. 671-679.

[21] R. Vilar, J. Zapata and R. Ruiz, "An Automatic System of Classification of Weld Defects in Radiographic Images", NDT \&E International, vol. 42, (2009), pp. 467-476.

[22] H. S. Cho and S. Rhee, "Experimental Study of Nugget Information in Resistance Spot Welding", Welding Journal, vol. 82, no. 4, (2003), pp. 195-200.

[23] W. Gan and T. W. Liao, "Automatic Identification of Different Types of Welding Defects in Radio graphic Images", Independent Nondestructive Testing and Evaluation International, vol. 35, no. 8, (2002), pp. 519-528.

[24] G. H. Y. Hang, "Combination of Feature-Based and Area-Based Image Registration Technique for High Resolution Remote Sensing Image", IEEE International Symposium on Geoscience and Remote Sensing, IGARSS, (2007) July 23-27, Barcelona, Spain.

[25] Z. H. Lin, Y. A. Zhang, G. U. Chen and Y. O. Li, "Study on Real-Time Measurement of Nugget Diameter for Resistance Spot Welding Using a Neuro-Fuzzy Algorithm", In Proceedings of the IEEE Instrumentation and Measurement Technology Conference (IMTC 2004), (2004) May 23-27, Como, Italy.

[26] S. A. Lee and Y. O. Choo, "A Quality Assurance Technique for Resistance Spot Welding Using A Neuro-Fuzzy Algorithm”, J Manuf Syst., vol. 20, no. 5, (2011), pp. 320-328.

[27] Y. O. Cho and S. E. Rhee, "Quality Estimation of Resistance Spot Welding by Using Pattern Recognition with Neural Networks", IEEE Trans Instrum Meas., vol. 53, no. 2, (2004), pp. 330-334. 


\section{Authors}

Yang Ou, has received a $\mathrm{PhD}$ in optical engineering from hust (Huazhong University of Science and Technology) in 2008. He has teaching experience of 7 years. Presently he is working as associate professor in Department of Applied Computer Engineering. His areas of interest are digital signal processing, computer vision, pattern recognition. 
International Journal of Signal Processing, Image Processing and Pattern Recognition Vol. 8, No. 5 (2015) 